\title{
REGULARIZED FIELDMAP ESTIMATION IN MRI
}

\author{
Jeffrey A. Fessler and Desmond Yeo \\ EECS Dept., The University of Michigan \\ \{fessler, tbyeo\}@umich.edu
}

\author{
Douglas C. Noll* \\ BME Dept., The University of Michigan \\ dnollaumich.edu
}

\begin{abstract}
In fast MR imaging with long readout times, such as echoplanar imaging (EPI) and spiral scans, it is important to correct for the effects of field inhomogeneity to reduce image distortion and blurring. Such corrections require an accurate field map, a map of the off-resonance frequency at each voxel. Standard fieldmap estimation methods yield noisy fieldmaps, particularly in image regions having low spin density. This paper describes regularized methods for fieldmap estimation. These methods exploit the fact that fieldmaps are smooth functions. Efficient convergent algorithms are given even though the problem is highly nonlinear. Results show that the proposed regularized methods significantly improve the quality of fieldmap estimates relative to conventional unregularized methods.
\end{abstract}

\section{INTRODUCTION}

For field-corrected MR image reconstruction, e.g., [1,2]: one must have available an accurate estimate of the fieldmap $\boldsymbol{\omega}=$ $\left(\omega_{1}, \ldots, \omega_{N}\right)$, where $N$ is the number of pixels. A common approach to measuring fieldmaps is to acquire two scans of the object with slightly different echo times, and then to reconstruct images $\boldsymbol{y}$ and $\boldsymbol{z}$ (without field correction) from those two scans. The usual model for those reconstructed images is

$$
\begin{aligned}
& y_{j}=f_{j}+\varepsilon_{j} \\
& z_{j}=f_{j} \mathrm{e}^{\imath \omega_{j} \triangle_{t}}+\eta_{j},
\end{aligned}
$$

where $\triangle_{t}$ denotes the echo-time difference, $f_{j}$ denotes the underlying complex transverse magnetization in the $j$ th voxel, and $\varepsilon_{j}$ and $\eta_{j}$ denote (complex) noise. The goal is to estimate $\boldsymbol{\omega}$ from $\boldsymbol{y}$ and $\boldsymbol{z}$, whereas $\boldsymbol{f}=\left(f_{1}, \ldots, f_{N}\right)$ is a nuisance parameter vector. For simplicity, we define the unknown phase to be $x_{j}=\omega_{j} \triangle_{t}$, so that the goal is to estimate $\boldsymbol{x}$ from $\boldsymbol{y}$ and $z$. The next section reviews the standard approach for this problem, and then describes three new and improved methods.

\footnotetext{
* Supported in part by NIH grants EB002683 and DA15410.
}

\section{FIELDMAP ESTIMATION METHOD}

\subsection{Conventional phase / fieldmap estimator}

The usual estimator $\hat{x}_{j}$ uses the phase difference of the two images, computed as follows [1]:

$$
\hat{x}_{j}=\angle\left(y_{j}^{*} z_{j}\right)=\angle z_{j}-\angle y_{j},
$$

and the fieldmap estimate is simply a scaled version: $\hat{\omega}_{j}=$ $\hat{x}_{j} / \triangle_{t}$. This expression would work perfectly in the absence of noise and phase wrapping, within voxels where $\left|f_{j}\right|>0$. However, (2) can be very sensitive to noise, particularly in voxels where the image magnitude $\left|f_{j}\right|$ is small relative to the noise deviations. Furthermore, that estimate ignores our a priori knowledge that fieldmaps tend to be smooth or piecewise smooth. Although one could try to smooth the above estimate using a lowpass filter, usually many of the $\hat{x}_{j}$ values are severely corrupted so smoothing will further propagate such errors. Instead, we propose below to integrate the smoothing into the estimation of $\boldsymbol{x}$ in the first place, rather than trying to "fix" the noise in $\hat{\boldsymbol{x}}$ by post processing.

\subsection{Maximum-likelihood phase / fieldmap estimation}

The conventional estimate (2) appears to disregard noise effects, so a natural approach is to estimate $\boldsymbol{x}$ using a maximum likelihood (ML) method based on a statistical model for the measurements $\boldsymbol{y}$ and $\boldsymbol{z}$. In MR, the $k$-space measurements have zero-mean white gaussian complex noise, and we furthermore assume that the additive noise values in $y$ and $z$ in (1) are independent and have the same variance $\sigma^{2}$. Under these assumptions, the joint $\log$-likelihood for $\boldsymbol{f}$ and $\boldsymbol{x}$ given $\boldsymbol{y}$ and $\boldsymbol{z}$ is

$$
\begin{aligned}
& \log \mathrm{p}(\boldsymbol{y} ; \boldsymbol{f})+\log \mathrm{p}(\boldsymbol{z} ; \boldsymbol{f}, \boldsymbol{x}) \\
& \quad \equiv \frac{-1}{2 \sigma^{2}} \sum_{j=1}^{N}\left|y_{j}-f_{j}\right|^{2}+\left|z_{j}-f_{j} \mathrm{e}^{\imath x_{j}}\right|^{2},
\end{aligned}
$$

where " $\equiv$ " denotes equality to within constants independent of $\boldsymbol{x}$. Thus, one achieves simultaneous ML estimation of $\boldsymbol{f}$ and $\boldsymbol{x}$ by the following minimization problem:

$$
\underset{\boldsymbol{x} \in \mathbb{R}^{N}}{\arg \min } \underset{\boldsymbol{f} \in \mathbb{C}^{N}}{\arg \min } \sum_{j=1}^{N}\left\|\left[\begin{array}{l}
y_{j} \\
z_{j}
\end{array}\right]-\left[\begin{array}{c}
1 \\
\mathrm{e}^{\imath x_{j}}
\end{array}\right] f_{j}\right\|^{2} .
$$


This problem is quadratic in $f_{j}$, leading to the following ML estimate:

$$
\hat{f}_{j}=\frac{y_{j}+\mathrm{e}^{-\imath x_{j}} z_{j}}{2} .
$$

Substituting this estimate back into the cost function and simplifying yields the following minimization problem for ML estimation of $\boldsymbol{x}$ :

$$
\underset{\boldsymbol{x}}{\arg \min } \Psi(\boldsymbol{x}), \quad \Psi(\boldsymbol{x})=\sum_{j=1}^{N} \frac{1}{2}\left|y_{j}-\mathrm{e}^{-\imath x_{j}} z_{j}\right|^{2} .
$$

Now note that

$$
\begin{aligned}
& \frac{1}{2}\left|y_{j}-\mathrm{e}^{-\imath x_{j}} z_{j}\right|^{2} \equiv-\frac{1}{2}\left(y_{j} \mathrm{e}^{\imath x_{j}} z_{j}^{*}+y_{j}^{*} \mathrm{e}^{-\imath x_{j}} z_{j}\right) \\
& \quad=-\operatorname{real}\left(y_{j}^{*} \mathrm{e}^{-\imath x_{j}} z_{j}\right)=-\left|y_{j}^{*} z_{j}\right| \cos \left(\angle z_{j}-\angle y_{j}-x_{j}\right) .
\end{aligned}
$$

Thus the ML cost function is equivalent to

$$
\Psi(\boldsymbol{x}) \equiv \sum_{j=1}^{N}\left|y_{j}^{*} z_{j}\right|\left[1-\cos \left(\angle z_{j}-\angle y_{j}-x_{j}\right)\right] .
$$

The ML estimate is not unique here due to the possibility of phase wrapping. But ignoring that issue, because $1-\cos (t)$ has a minimum at zero, the ML estimate of $\boldsymbol{x}$ is $\hat{x}_{j}=\angle z_{j}-$ $\angle y_{j}$, which is simply the usual estimate (2) once again. Thus the usual method is in fact an ML estimator under the white gaussian noise model!

\subsection{Penalized likelihood phase / fieldmap estimation}

The ML estimator ignores our a priori knowledge that fieldmaps tend to be spatially smooth functions due to the physical nature of main field inhomogeneity and susceptibility effects. A natural approach to incorporating this characteristic is to add a regularizing roughness penalty to the cost function. Here we regularize only the phase map $\boldsymbol{x}$ and not the magnetization map $\boldsymbol{f}$; we expect $\boldsymbol{f}$ to be far less smooth because it contains anatomical details. Such regularization is equivalent to replacing ML estimation with a certain Bayesian MAP estimator. In either case, based on (3) the resulting regularized cost function has the form

$$
\Psi(\boldsymbol{x})=\sum_{j=1}^{N}\left|y_{j} z_{j}\right|\left[1-\cos \left(\angle z_{j}-\angle y_{j}-x_{j}\right)\right]+\beta R(\boldsymbol{x}),
$$

where $R(\boldsymbol{x})$ is a spatial roughness penalty. This cost function automatically gives low weight to any voxels where the magnitude $\left|y_{j} z_{j}\right|$ is small. For such voxels, the regularization term will have the effect of smoothing or extrapolating the neighboring values. Thus, this approach avoids the phase "outlier" problem that plagues the usual estimate (2) in voxels with low signal magnitude.

If $\boldsymbol{x}$ corresponds to a $N \times M$ fieldmap $x[n, m]$, then a typical regularizing roughness penalty uses the differences between horizontal and vertical neighboring voxel values as follows:

$$
\begin{aligned}
R(\boldsymbol{x}) & =\sum_{n=1}^{N-1} \sum_{m=0}^{M-1} \psi(x[n, m]-x[n-1, m]) \\
& +\sum_{n=0}^{N-1} \sum_{m=1}^{M-1} \psi(x[n, m]-x[n, m-1]) .
\end{aligned}
$$

Usually $\psi$ is differentiable, so we can minimize the cost function $\Psi(\boldsymbol{x})$ either by conventional gradient descent methods or by optimization transfer methods [3]. In particular, in the usual case where $\dot{\psi}(t) / t$ is bounded by unity, then one can show that the following iteration is guaranteed to decrease $\Psi(\boldsymbol{x})$ monotonically:

$$
\boldsymbol{x}^{(n+1)}=\boldsymbol{x}^{(n)}-\operatorname{diag}\left\{\frac{1}{\left|y_{j} z_{j}\right|+\beta \cdot 4}\right\} \nabla \Psi\left(\boldsymbol{x}^{(n)}\right),
$$

because the second derivative of $1-\cos t$ is bounded above by unity. This algorithm will converge to a local minimizer of $\Psi(\boldsymbol{x})$ within the "basin" that contains the initial estimate [3]. We use the ML estimate to initialize $\boldsymbol{x}^{(0)}$.

\subsection{PWLS fieldmap estimator}

Usually the time delay $\triangle_{t}$ is chosen to ensure that there will be little if any phase wrapping. In such cases, we can simplify computation by approximating the $1-\cos$ term in (4) with its second-order Taylor series: $1-\cos (t) \approx t^{2} / 2$. Substituting this approximation into (4) leads to the following penalized weighted least squares (PWLS) cost function for estimating $\boldsymbol{x}$ :

$$
\Psi(\boldsymbol{x})=\sum_{j=1}^{N} w_{j} \frac{1}{2}\left(\angle z_{j}-\angle y_{j}-x_{j}\right)^{2}+\beta R(\boldsymbol{x}),
$$

where we define a magnitude weighting function as follows:

$$
w_{j} \triangleq\left|y_{j} z_{j}\right| \text {. }
$$

PWLS estimators give more weight to the "good data" and use regularization to control noise.

In the current image processing literature, edge-preserving potential functions $\psi$ are quite popular. However, because fieldmaps are spatially smooth and often free of "edges," typically we use the quadratic function $\psi(t)=t^{2} / 2$ here. Because in this case the cost function (7) is quadratic, it is minimized easily by the conjugate-gradient (CG) algorithm. We denote the resulting method as QPWLS-CG. If one encounters fieldmaps that have steep gradients, then it may be preferable to use an edge-preserving potential function $\psi$. In such cases one can use a CG algorithm with a modified line search [4] to minimize $\Psi$ efficiently. However, the model (1) may be unrealistic if the field gradients are so steep that there is substantial within-voxel variation. For such cases it may be 
necessary to consider other formulations such as the k-space approach described in $\S 2.6$.

To simplify processing somewhat further, one could binarize the weights $w_{j}$ using a threshold:

$$
w_{j} \triangleq \begin{cases}1, & \left|y_{j} z_{j}\right|>\gamma \\ 0, & \text { otherwise }\end{cases}
$$

where we set the threshold $\gamma$ to include only voxels with "sufficiently large" magnitude, e.g., $\gamma=0.4 \max _{j}\left|y_{j} z_{j}\right|$. This approach was used routinely in our group prior to the development of the ML-based weighting in (7).

The primary limitation on of the cost function (7) is that it ignores any phase wrap that may occur when evaluating (2). If such phase wrap is possible, then it may be preferable to use the penalized likelihood estimator (4).

\subsection{Example}

Fig. 1 shows an example of the data magnitude $\left|y_{j}\right|$ and the usual phase estimate (2) which is very noisy. This is real data taken from a 3T MR scanner. It also shows the penalized likelihood estimate based on (6), the QPWLS estimate from (7), and the QPWLS estimate using (9). The normalized RMS difference between the penalized likelihood method and the QPWLS approximation (7) was 3.1\%, whereas the QPWLS results based on the binary weights (9) differed by over $40 \%$ normalized RMS. Compared to penalized-likelihood, the QPWLS cost function is somewhat easier to minimize, so QPWLS with the approximation (7) is a practical and accurate approach in the usual cases where phase wrapping is not expected.
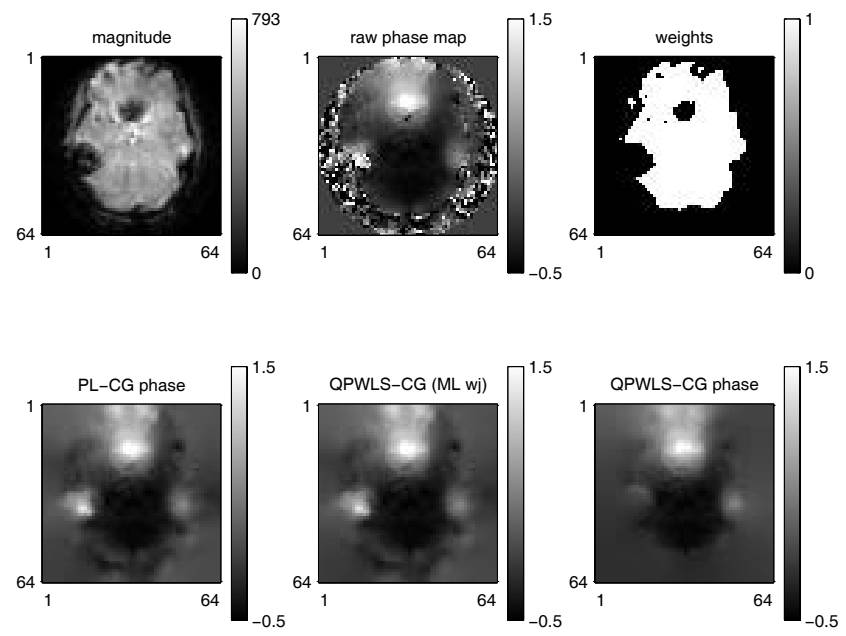

Fig. 1. Top row: magnitude image $\left|y_{j}\right|$, usual phase estimate (2), and binary weights $w_{j}$ in (9). Bottom row (phase estimates): penalized likelihood using (6), QPWLS using (7), and QPWLS using (9).

\subsection{Fieldmap estimation in k-space}

The methods described above estimate the fieldmap from two reconstructed images. To work well, those images should be relatively free of artifacts, blur, and distortions, necessitating appropriate data acquisition types. For pulse sequences with long readout times, it may be more appropriate to estimate the fieldmap directly from the raw k-space data. A typical scenario is that we can collect two sets of k-space data, with slightly different echo times, from which we want to estimate the fieldmap $\boldsymbol{\omega}$ and the baseline magnetization $f$. A reasonable model for the data is:

$\mathrm{E}\left[y_{i}^{(l)}\right]=\int f(\overrightarrow{\mathrm{x}}) \mathrm{e}^{-\imath \omega(\overrightarrow{\mathrm{x}})\left(t_{i}+l \triangle_{t}\right)} \mathrm{e}^{-\imath 2 \pi \vec{\nu}_{i} \cdot \overrightarrow{\mathrm{x}}} \mathrm{d} \overrightarrow{\mathrm{x}}, l=0,1$.

This is a joint estimation problem like that described in [5]. One can define a cost function in terms of $\boldsymbol{f}$ and $\boldsymbol{\omega}$, and then alternate between holding $\boldsymbol{\omega}$ fixed and minimizing over $\boldsymbol{f}$ (using the CG method) and then holding $f$ fixed and minimizing over $\boldsymbol{\omega}$ (using steepest descent [5] or linearization [6] or optimization transfer methods akin to [7]). These k-space methods require considerably more computation than the image domain methods, so one should first apply an image-domain method to get a reasonable initial estimate of the fieldmap $\omega$.

\subsection{Spatial resolution analysis of fieldmap estimation}

One drawback of the regularized methods (4) and (7) above is that the user must select the regularization parameter $\beta$, which could seem tedious if one uses trial-and-error methods. Fortunately, it is particularly simple to analyze the spatial resolution properties for this problem, using the methods in [8] for example. The local frequency response of the QPWLS estimator (7) at the $j$ th voxel can be shown to be

$$
H\left(\omega_{1}, \omega_{2}\right) \approx \frac{1}{1+\left(\beta / w_{j}\right)\left(\omega_{1}^{2}+\omega_{2}^{2}\right)^{p}},
$$

where $p=1$ for regularization based on first-order differences as in (5), and $p=2$ for 2 nd-order finite differences. (See [9] for related analyses.) Using this form, one can use the inverse 2D DSFT to compute the PSF $h[n, m]$ and tabulate its FWHM as a function of $\beta / w_{j}$. Fig. 2 shows this FWHM as a function of $\log _{2}\left(\beta / w_{j}\right)$, for both $p=1$ and $p=2$. The FWHM increases monotonically with $\beta$, as expected, although the "knees" in the curve are curious. Nevertheless, one can use this graph to select the appropriate $\beta$ given the desired spatial resolution in the estimated fieldmap. To simplify such selection, we normalize the weights in (8) by the median of the nonzero values so that the "typical" $w_{j}$ value is unity. The resulting spatial resolution will be inherently nonuniform, with more smoothing in the regions with low magnitudes $w_{j}$ and vice versa. One could explore modified regularization methods [8] to make the resolution uniform, but in this application nonuniform resolution seems appropriate since the goals include "interpolating" across signal voids. 
Fig. 3 shows that the shape of the PSF depends strongly on whether one uses regularization based on 1st-order or 2ndorder finite differences. These profiles suggest that 2nd-order differences are preferable since the PSF tails decrease more rapidly even when the FWHM values are identical.

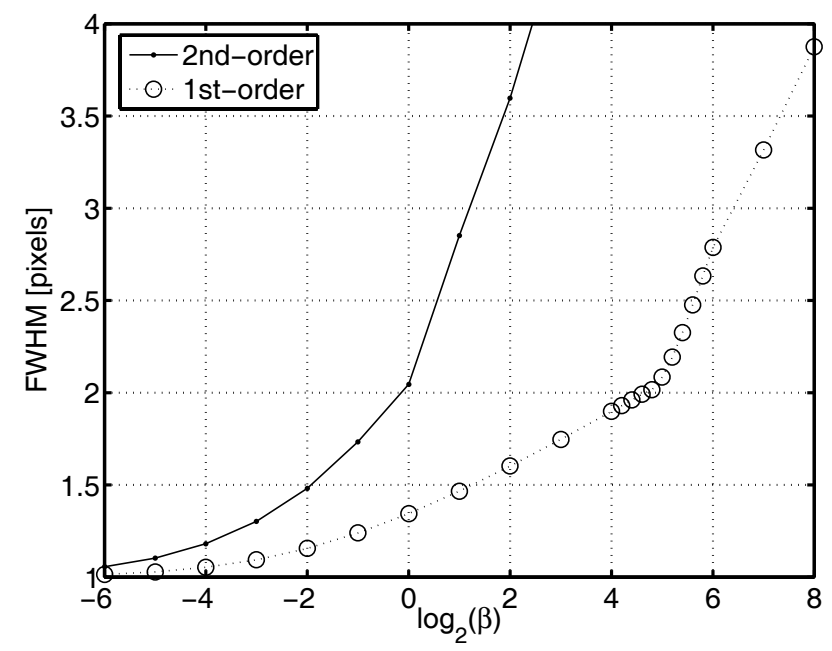

Fig. 2. Angularly averaged FWHM of PSF for fieldmap estimation as a function of $\log _{2} \beta$ for $w_{j}=1$.

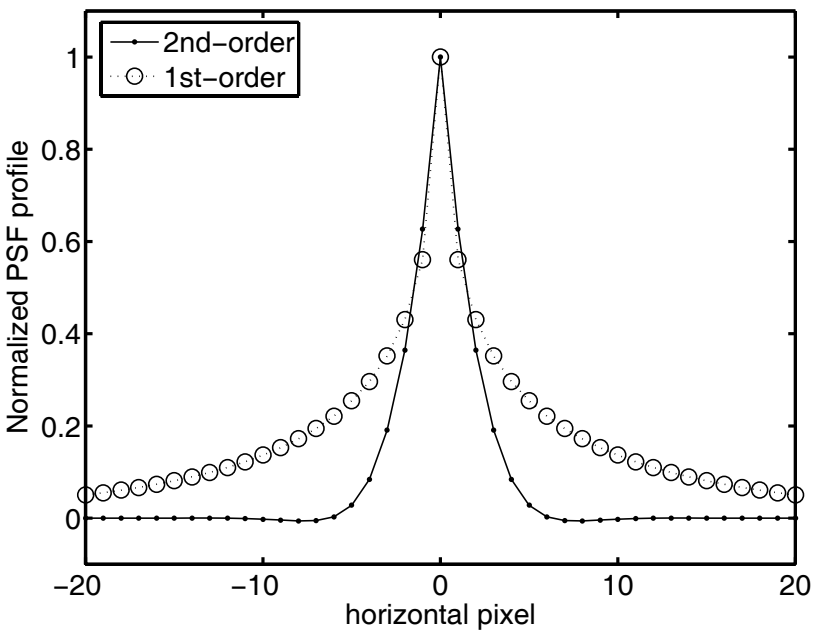

Fig. 3. Profiles through reconstructed PSF corresponding to (10) for regularization based on 1st-order or 2nd-order finite differences. The regularization parameter $\beta$ was chosen in both cases so that the FWHM was 3 pixels.

\section{DISCUSSION}

We have described two regularized methods for fieldmap estimation, the penalized-likelihood method (4) and the PWLS method (7). Both of these methods yield fieldmaps that interpolate smoothly over regions with low spin density, thereby avoiding the phase outliers that plague the conventional estimate (2).

Our analysis also shows that the conventional estimate (2) is in fact the ML estimate, a property that has previously gone unnoticed to our knowledge.

We also analyzed the spatial resolution properties of these regularized methods, leading to a practical procedure for choosing the regularization parameter to achieve a given desired spatial resolution.

It is desirable to show how the field maps shown in Fig. 1 will affect the image quality for standard trajectories such as EPI or a spiral. Space constraints prohibit such images here, but will be shown in a longer version in preparation.

\section{REFERENCES}

[1] K. Sekihara, S. Matsui, and H. Kohno, "NMR imaging for magnets with large nonuniformities," IEEE Trans. Med. Imag., vol. 4, no. 4, pp. 193-9, Dec. 1985.

[2] B. P. Sutton, D. C. Noll, and J. A. Fessler, "Fast, iterative image reconstruction for MRI in the presence of field inhomogeneities," IEEE Trans. Med. Imag., vol. 22, no. 2, pp. 178-88, Feb. 2003.

[3] M. W. Jacobson and J. A. Fessler, "Properties of MM algorithms on convex feasible sets: extended version," Tech. Rep. 353, Comm. and Sign. Proc. Lab., Dept. of EECS, Univ. of Michigan, Ann Arbor, MI, 48109-2122, Nov. 2004.

[4] J. A. Fessler and S. D. Booth, "Conjugate-gradient preconditioning methods for shift-variant PET image reconstruction," IEEE Trans. Im. Proc., vol. 8, no. 5, pp. 688-99, May 1999.

[5] B. P. Sutton, D. C. Noll, and J. A. Fessler, "Dynamic field map estimation using a spiral-in / spiral-out acquisition," Mag. Res. Med., vol. 51, no. 6, pp. 1194-204, June 2004.

[6] V. Olafsson, J. A. Fessler, and D. C. Noll, "Dynamic update of R2* and field map in fMRI," in Proc. Intl. Soc. Mag. Res. Med., 2004, p. 45.

[7] J. A. Fessler and D. C. Noll, "Iterative image reconstruction in MRI with separate magnitude and phase regularization," in Proc. IEEE Intl. Symp. Biomed. Imag., 2004, pp. 209-12.

[8] J. A. Fessler and W. L. Rogers, "Spatial resolution properties of penalized-likelihood image reconstruction methods: Spaceinvariant tomographs," IEEE Trans. Im. Proc., vol. 5, no. 9, pp. 1346-58, Sept. 1996.

[9] M. Unser, A. Aldroubi, and M. Eden, "Recursive regularization filters: design, properties, and applications," IEEE Trans. Patt. Anal. Mach. Int., vol. 13, no. 3, pp. 272-7, Mar. 1991. 